TU-935

\title{
Suppressing Isocurvature Perturbations of QCD Axion Dark Matter
}

\author{
Kwang Sik Jeong* and Fuminobu Takahashi ${ }^{\dagger}$ \\ Department of Physics, Tohoku University, Sendai 980-8578, Japan
}

\begin{abstract}
We propose a novel mechanism to suppress the isocurvature perturbations of the QCD axion. The point is that the QCD interactions become strong at an intermediate or high energy scale in the very early Universe, if the Higgs field has a sufficiently large expectation value. The effective QCD scale can be even higher in the presence of extra colored particles. We show that the QCD axion then becomes so heavy during inflation that its isocurvature perturbations are significantly suppressed, thereby relaxing the constraint on the inflation scale.
\end{abstract}

The axion $a$ is a Nambu-Goldstone boson associated with the spontaneous breakdown of the Peccei-Quinn (PQ) symmetry introduced to solve the strong CP problem in quantum chromodynamics (QCD) [1, 2]. The axion acquires a tiny but non-zero mass from the QCD instanton effects, and it is stabilized at the $\mathrm{CP}$ conserving vacuum. The dynamical relaxation of the $\mathrm{CP}$ phase necessarily induces coherent oscillations of the axion, which contribute to cold dark matter (CDM) as the axion is stable in a cosmological time scale.

If the axion is present during inflation, it is subject to quantum fluctuations,

$$
\delta a \simeq \frac{H_{\mathrm{inf}}}{2 \pi}
$$

where $H_{\text {inf }}$ denotes the Hubble parameter during inflation. The quantum fluctuations lead to an almost scale-invariant CDM isocurvature density fluctuation, which leaves a distinctive imprint on the cosmic microwave background (CMB) spectrum. The observed CMB spectrum can be well fitted by a nearly scale-invariant adiabatic density perturbation, and a mixture of the isocurvature perturbation is tightly constrained. This constraint can be interpreted as an upper bound on the inflation scale in the axion CDM scenario [3],

$$
H_{\text {inf }}<0.87 \times 10^{7} \mathrm{GeV}\left(\frac{f_{a}}{10^{11} \mathrm{GeV}}\right)^{0.408}
$$

at $95 \% \mathrm{CL}$, where $f_{a}$ is the axion decay constant [4, 5]. The constraint becomes much more

\footnotetext{
*email: ksjeong@tuhep.phys.tohoku.ac.jp

$\dagger$ email: fumi@tuhep.phys.tohoku.ac.jp
}

stringent for a smaller value of $f_{a}$ due to anharmonic effects [6, 7]. Clearly, a chaotic inflation model [8 11] is in tension with the axion CDM.

There are several known ways to avoid the constraint. First, if the PQ symmetry is restored during inflation, the axion does not exist, and so there is no isocurvature perturbation [12, 13]. The axion appears when the PQ symmetry is spontaneous broken after inflation. In such case, however, topological defects such as cosmic strings and domain walls are generated, and in particular the domain wall number must be unity to avoid the overclosure of the Universe. Secondly, if the coefficient of the axion kinetic term was larger during inflation than at present, the size of $\delta a$ can be suppressed. This can be realized if the radial component of the $\mathrm{PQ}$ scalar has a flat potential and takes a larger value during inflation [12, 14]. Alternatively, a similar effect is possible if there is a non-minimal coupling to gravity [15].

In this letter we propose another way to relax the isocurvature constraint on the inflation scale in the axion CDM scenario. The point is that, if the Higgs field has a sufficiently large expectation value during inflation, the QCD confines at an intermediate or high energy scale because the quarks decouple at a high energy scale and make the QCD coupling run faster at lower scales. Then the axion becomes so heavy that its quantum fluctuations at superhorizon scales are significantly suppressed, thereby relaxing the constraint on the inflation scale. The QCD scale can be even higher in the presence of additional colored particles that become heavy during inflation.

A stronger QCD in the early Universe was considered in Refs. [16, 17] in order to suppress the cosmological abundance of axions produced by the misalignment mechanism, which however turned 
out rather non-trivial to be realized [18]. We will show that the stronger QCD can suppress the axion quantum fluctuations, but not the abundance in general.

There are a variety of scenarios leading to a large field value of the Higgs fields [19 21]. This can naturally be realized in the supersymmetric (SUSY) standard model (SM) which possesses flat directions involving the Higgs fields $H_{u}$ and $H_{d}$. In this letter we consider a SUSY axion model, but our main idea can be applied to the non-SUSY case as well as other string theoretic axions in a straightforward way. In the following we adopt the Planck unit in which $M_{P l} \simeq 2.4 \times 10^{18} \mathrm{GeV}$ is set to be unity.

We now examine how heavy the axion can be during inflation when the $H_{u} H_{d}$ flat direction has a large field value. In the following we assume that the Higgs fields are neutral under the PQ symmetry. ${ }^{1}$ The relevant scales are the axion decay constant $f_{a}$, the effective QCD scale $\Lambda_{h}$, and the inflation scale $H_{\text {inf }}$. For simplicity we assume that the Kähler potential is a generic one such that soft scalar masses are of order the gravitino mass $m_{3 / 2}$, and that $f_{a}$ remains constant during and after inflation.

Let $\phi^{2}$ parameterize the $H_{u} H_{d}$ flat direction. It gets a mass correction, $-c H_{\text {inf }}^{2}|\phi|^{2}$, from a Plancksuppressed quartic coupling with the inflaton $X$ in the Kähler potential. The Hubble-induced mass term drives $\phi$ to a large field value for positive $c$ if $H_{\text {inf }}>m_{3 / 2}$. Then the flat direction is stabilized at $\phi_{0} \simeq c^{\frac{1}{4}} \sqrt{H_{\text {inf }} M}$ by the higher order superpotential term,

$$
\Delta W=\frac{\phi^{4}}{4 M},
$$

where $M$ is an effective cut-off scale. We will assume $\phi_{0}$ to be around or above the GUT scale, $M_{\mathrm{GUT}} \simeq 2 \times 10^{16} \mathrm{GeV}$, which can be realized for an appropriate value of $M$. It is also possible to stabilize $\phi$ by a higher order term in the Kähler potential. Note that the mass of the radial component of $\phi$ is of order $H_{\text {inf }}$. The phase component

1 If $H_{u}$ and $H_{d}$ are charged under the PQ symmetry, the effective axion decay constant during inflation is given by $\phi_{0}$. The isocurvature perturbation constraint can be similarly relaxed, if one introduces a singlet which gives a mass to additional quarks. of $\phi$ can have a mass of similar size in the presence of interactions like $X|\phi|^{2}$ or $|X|^{2} \phi^{2}$ in the Kähler potential. In this case there are no light degrees of freedom associated with the flat direction. ${ }^{2}$

The SUSY SM matter fields including neutrinos acquire large SUSY mass from the Yukawa couplings with $H_{u}$ or $H_{d}$. Let us first consider a case in which all the quarks are heavier than $\Lambda_{h}$. One can then integrate them out and obtain a pure SUSY $\mathrm{SU}(3)_{c}$ with the gauge kinetic function [22],

$$
f_{h}=(\text { constant })-\frac{n}{8 \pi^{2}} \ln S-\frac{N_{f}}{8 \pi^{2}} \ln \phi,
$$

where $N_{f}=6$ is the flavor number of the SM quarks, and $S$ denotes the axion superfield which includes the axion,

$$
a=\sqrt{2}\langle|S|\rangle \arg (S) \equiv \frac{f_{a}}{n} \arg (S) .
$$

The constant $n$ counts the number of PQ charged quarks when the PQ symmetry is linearly realized. If $\Lambda_{h}$ is larger than $H_{\mathrm{inf}}$, the gluino condensate is formed during inflation, giving rise to the nonperturbative superpotential [23],

$$
\begin{aligned}
W_{\mathrm{np}} & =N_{c} \Lambda_{0}^{3} \propto e^{-8 \pi^{2} f_{h} / N_{c}} \\
& =(\text { constant }) \times S^{n / N_{c}} \phi^{N_{f} / N_{c}},
\end{aligned}
$$

where $N_{c}=3$, and $\Lambda_{0}$ denotes the condensation scale which is equal to $\Lambda_{h}$ in the present case. ${ }^{3}$ The scalar potential of $\phi$ is not affected significantly by $W_{\text {np }}$ as long as $H_{\text {inf }} \phi_{0}^{2} \gg \Lambda_{0}^{3}$, which is satisfied for the parameters of our interest. Also, $f_{a}>\Lambda_{0}$ is needed in order not to destabilize the saxion, as shown below. Then an axion potential is generated through the non-perturbative superpotential and SUSY breaking, with the dominant contribution from the Higgs $F$-term:

$$
\begin{aligned}
V & =\left|\partial_{\phi}\left(\Delta W+W_{\mathrm{np}}\right)\right|^{2}+\cdots \\
& =2 \sqrt{c} N_{f} H_{\mathrm{inf}} \Lambda_{0}^{3} \cos \left(\frac{a}{N_{c} f_{a}}+\theta_{0}\right)+\cdots,
\end{aligned}
$$

2 If the phase of $\phi$ is lighter than $H_{\text {inf }}$, it acquires quantum fluctuations, giving rise to the axion isocurvature perturbations with an amplitude suppressed by a factor of $f_{a} / \phi_{0}$ compared to the conventional scenario.

3 The Higgs $F$-term $F_{\phi} / \phi \simeq \sqrt{c} H_{\text {inf }}$ induces a gluino mass via gauge mediation. Thus $H_{\mathrm{inf}}$ should be smaller than $\Lambda_{0}$ for the gluino not to decouple at a scale above $\Lambda_{0}$. 
for the condensation scale in the range

$$
H_{\mathrm{inf}}<\Lambda_{0}<f_{a} .
$$

Here $\theta_{0}$ is generally different from the QCD angle since it receives various contributions including the phase of $\phi$. The axion mass thus reads

$$
m_{a}^{2}=\frac{\hat{c} H_{\mathrm{inf}} \Lambda_{0}^{3}}{f_{a}^{2}},
$$

with $\hat{c} \equiv 2 \sqrt{c} N_{f} / N_{c}^{2}$. Note that a constant term $m_{3 / 2} M_{P l}^{2}$ in the superpotential also contributes to the axion mass, which is important when $m_{3 / 2}>$ $H_{\text {inf }}$.

On the other hand, if there are $n_{f}$ quarks lighter than $\Lambda_{h}$, they form a meson field stabilized by balancing the Affleck-Dine-Seiberg potential and the SUSY mass term $m$. In practice, this is the case for $\Lambda_{h} \gtrsim 10^{11} \mathrm{GeV}$, where the up and down quarks are lighter than $\Lambda_{h}$. Integrating out the meson fields, one is left with the gluino condensation (6) with $\Lambda_{0}^{3 N_{c}}=\Lambda_{h}^{3 N_{c}-n_{f}} \operatorname{det}(m)$. Using $\Lambda_{0}$ defined this way, the axion mass formula (9) holds also in such cases.

The higher QCD scale and the heavy axion mass during inflation provide us with a simple way to suppress the axion CDM isocurvature perturbations. The axion mass is heavier than the inflation scale, i.e., $m_{a}>H_{\mathrm{inf}}$, if

$$
H_{\mathrm{inf}}<10^{12} \mathrm{GeV} \times \hat{c}\left(\frac{\Lambda_{0}}{f_{a}}\right)^{3}\left(\frac{f_{a}}{10^{12} \mathrm{GeV}}\right) .
$$

Then the axion is stabilized at one of the minima of the potential (7), and no sizable quantum fluctuations are generated at superhorizon scales. One can see that the isocurvature constraint on the inflation scale can be significantly relaxed compared to the conventional scenario (2).

So far we have assumed that $f_{a}$ remains the same during and after inflation. This is the case if the saxion stabilization is not significantly modified by the strong QCD interactions, which generically requires $\Lambda_{0}<f_{a}$. To see this, consider a model where the PQ symmetry is broken by two PQ fields $S_{1}$ and $S_{2}$ with the superpotential $W=\Sigma\left(S_{1} S_{2}-f_{0}^{2}\right)$ where $\Sigma$ is a PQ singlet. For $\Lambda_{0}<f_{0}$, the minimum will lie along the $F$ flat direction $S_{1} S_{2}=f_{0}^{2}$ while giving $f_{a} \sim f_{0}$, if $S_{1}$ and $S_{2}$ have soft scalar masses of similar size and couple to PQ charged quarks. On the other

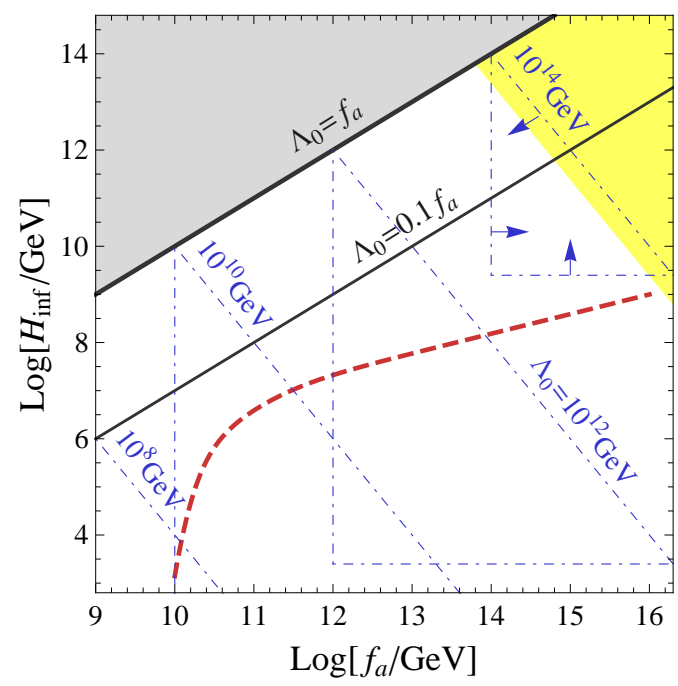

FIG. 1: The axion CDM isocurvature perturbation is suppressed by a heavy axion mass in the white region. See the text for explanation.

hand, the saxion potential is significantly affected for $\Lambda_{0}>f_{0}$, as the non-perturbative superpotential gives the dominant contribution to the saxion potential. Depending on the axion model, $f_{a}$ may have a different value from the present one even if $\Lambda_{0}<f_{a}$. However the suppression mechanism works as long as the QCD scale is high enough to satisfy the condition (10) for the value of $f_{a}$ during inflation.

The saxion can be copiously produced by coherent oscillations if its position during inflation is deviated from the low-energy potential minimum. If it dominates the energy density of the Universe, axions may be overproduced by the saxion decay. This can be avoided by e.g. introducing a certain coupling of the saxion to Higgs multiplet [24]. In fact, the saxion abundance depends on the details of the stabilization of the PQ fields. In the example considered above, the saxion coherent oscillations after inflation can be suppressed if there is an approximate symmetry under which $S_{1}$ and $S_{2}$ are interchanged.

Fig. 1 1 shows which region in the $\left(f_{a}, H_{\text {inf }}\right)$ plane is allowed by the constraint $m_{a}>H_{\text {inf }}$, where we take $\hat{c}=1$ and $\Lambda_{0}$ as a free parameter. We will consider an explicit model to realize a large value of $\Lambda_{0}$ later. In the upper-left shaded (gray) region, the saxion stabilization is significantly mod- 
ified by the QCD interactions as $\Lambda_{0}$ is larger than $f_{a}$. The thick (thin) solid line corresponds to the upper bound on $H_{\text {inf }}$ for $\Lambda_{0}=f_{a}\left(\Lambda_{0}=0.1 f_{a}\right)$. The upper-right yellow region is excluded by the perturbativity limit of the gauge interactions up to the GUT scale (16). Note that $m_{a}>H_{\text {inf }}$ requires the axion decay constant and the QCD scale during inflation to satisfy the condition (10), which is derived for $H_{\mathrm{inf}}>\Lambda^{3} / \phi_{0}^{2}$ and $H_{\mathrm{inf}}<\Lambda_{0}<f_{a}$. Thus, for fixed $\Lambda_{0}$, the axion CDM isocurvature perturbation is suppressed inside a right-angled triangle, whose base, height, and hypotenuse correspond to $\Lambda_{0}<f_{a}, H_{\mathrm{inf}}>\Lambda^{3} / \phi_{0}^{2}$ with $\phi_{0} \sim M_{\mathrm{GUT}}$, and $m_{a}>H_{\text {inf }}$, respectively. For instance, the upperright one with blue dot-dashed sides is obtained for $\Lambda_{0}=10^{14} \mathrm{GeV}$. We also show other cases with $\Lambda_{0}=10^{8}, 10^{10}, 10^{12} \mathrm{GeV}$, from left to right. The conventional isocurvature bound (2) is shown by the dashed red line, where the anharmonic effect [7] has been taken into account.

Let us here comment on the axion relic abundance from the misalignment mechanism. After inflation the axion is located at the minimum of the potential (7), which is generally different from the $\mathrm{CP}$ conserving one. Hence the axion dark matter abundance today reads [2]

$$
\Omega_{a} h^{2} \simeq 0.2 \theta_{i}^{2}\left(\frac{f_{a}}{10^{12} \mathrm{GeV}}\right)^{1.184}
$$

with the initial misalignment angle given by

$$
\theta_{i}=\theta_{0}-\theta_{\mathrm{QCD}}
$$

where $\theta_{\mathrm{QCD}}$ denotes the QCD angle. For $f_{a} \gg$ $10^{12} \mathrm{GeV},\left|\theta_{i}\right| \ll 1$ is needed to avoid overclosure of the Universe. In other words, the effect of the stronger QCD is to suppress the axion quantum fluctuations, not the abundance.

The QCD confines at a high energy scale if the Higgs fields are stabilized at a large field value during inflation, because the quarks obtain large masses. A similar effect occurs if there are extra colored particles that become heavier during inflation. Let us add $N_{\Psi}$ pairs of $\Psi+\Psi^{c}$ that belong to $\mathbf{5}+\overline{\mathbf{5}}$ of $\mathrm{SU}(5)$ but do not carry PQ charges:

$$
W=\left(M_{\Psi}+\frac{\phi^{2}}{M^{\prime}}\right) \Psi \Psi^{c},
$$

They are fixed at the origin if $M_{\Psi}+\phi^{2} / M^{\prime}$ is larger than $m_{3 / 2}$ and $H_{\text {inf }}$. For $\phi$ stabilized around the
GUT scale, $\Psi+\Psi^{c}$ become heavy, and therefore the QCD confines at a larger scale than in the MSSM. Such effect is maximized when the effective cut-off scale $M^{\prime}$ is around $M_{\mathrm{GUT}}$. We find that the $\mathrm{QCD}$ scale can be as high as,

$$
\Lambda_{h} \simeq 1.3 \times 10^{7} \mathrm{GeV}\left(\frac{M_{\mathrm{GUT}}}{M_{\Psi}}\right)^{N_{\Psi} / 9},
$$

for $\phi_{0} \sim M^{\prime} \sim M_{\mathrm{GUT}}$. The above result shows that $\Lambda_{h}$ is about $10^{7} \mathrm{GeV}$ when the SM quarks obtain heavy masses from a large Higgs value around the GUT scale, but it can be raised much higher in the presence of extra colored particles. Here we have taken into account the hierarchy in the SM quark masses, and have used that the universal gauge coupling constant at the GUT scale is $g_{\mathrm{GUT}}^{2} \simeq 0.5$ in the MSSM.

It is also important to note that $\Psi+\Psi^{c}$ increase the axion mass since they decouple while giving a contribution to the coefficient of $\ln \phi$ in $f_{h}$ in the effective theory. It is straightforward to see that $\hat{c}$ is enhanced by a factor $1+2 N_{\Psi} / N_{f}$, and therefore the constraint on $H_{\text {inf }}$ is further relaxed.

On the other hand, in the axion models where the PQ symmetry is linearly realized, there should exist PQ charged quarks in order for $S$ to couple to the QCD anomaly. Let us introduce $N_{\Phi}$ pairs of $\Phi+\Phi^{c}$ transforming as $\mathbf{5}+\overline{\mathbf{5}}$ under $\mathrm{SU}(5)$, and consider the superpotential term $S \Phi \Phi^{c}$. Then $\Phi+\Phi^{c}$ obtain large masses $M_{\Phi} \sim f_{a}$. The gauge coupling unification is maintained in the presence of additional $\mathbf{5}+\overline{\mathbf{5}}$ matter fields, but the gauge coupling at the GUT scale is larger than the MSSM value. In order for the gauge interactions to remain perturbative up to $M_{\mathrm{GUT}}$, we need

$$
\frac{1}{g_{\mathrm{GUT}}^{2}} \simeq 2-\sum_{i=\Phi, \Psi} \frac{N_{i}}{8 \pi^{2}} \ln \left(\frac{M_{\mathrm{GUT}}}{M_{i}}\right)>\frac{1}{4 \pi} \text {. }
$$

Note that the PQ charged quarks play no role in raising the QCD scale because their masses remain the same during and after inflation. The above requirement is combined with the relation (14) to put the upper bound on the QCD scale,

$$
\Lambda_{h}<2.7 \times 10^{14} \mathrm{GeV}\left(\frac{M_{\Phi}}{M_{\mathrm{GUT}}}\right)^{N_{\Phi} / 9},
$$

which is insensitive to $M_{\Psi}$. Thus the QCD scale can be high enough to achieve $m_{a}>H_{\text {inf }}$ in the early Universe. For instance, for $M_{\Phi} \geq 10^{9} \mathrm{GeV}$ 


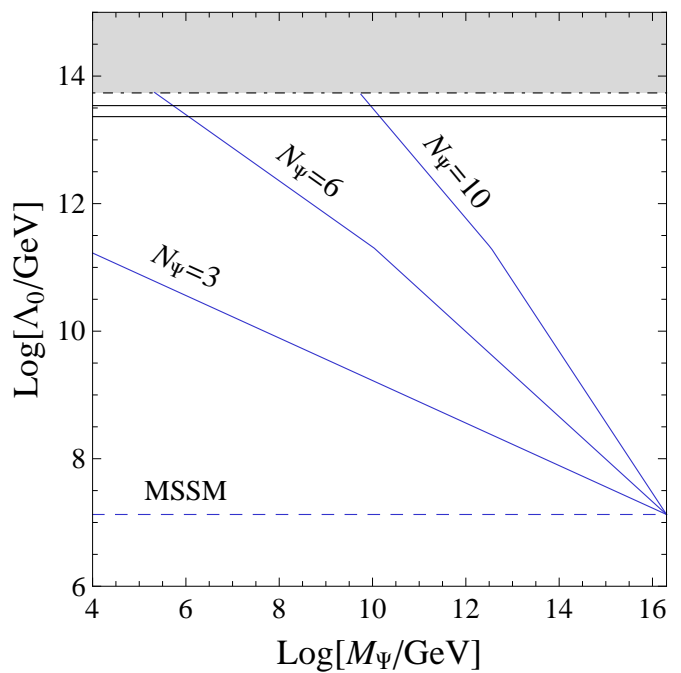

FIG. 2: Condensate scale $\Lambda_{0}$ for $\phi$ fixed at $M_{\mathrm{GUT}}$ in the presence of extra $\mathbf{5}+\overline{\mathbf{5}}$ matter fields. See the text for explanation.

and $N_{\Psi}=1, \Lambda_{0}$ can be as high as about $10^{13}$ $\mathrm{GeV}$. We note that $\Lambda_{0}$ is slightly smaller than $\Lambda_{h}$ if $\Lambda_{h} \gtrsim 10^{-5} M_{\mathrm{GUT}}$, due to the hierarchy of the SM quark masses.

Fig. 2 shows how much $\Lambda_{0}$ can be raised for $\phi_{0}=M_{\mathrm{GUT}}$ during inflation, under the requirement of the perturbativity of gauge couplings up to the GUT scale. The solid (blue) lines represent $\Lambda_{0}$ for $N_{\Psi}=3,6,10$ from bottom to top, while the dashed one is for the MSSM without extra matter fields. In the shaded region above the dotdashed black line, the perturbativity of gauge couplings breaks down below $M_{\mathrm{GUT}}$. This bound becomes severer in the presence of PQ charged fields $\Phi+\Phi^{c}$ : the upper (lower) solid-black line is the bound for the case with $N_{\Phi}=1$ and $M_{\Phi}=10^{14}$ $\mathrm{GeV}\left(M_{\Phi}=10^{12} \mathrm{GeV}\right)$.

The axion mass depends on $c$, the coupling of $\phi$ with the inflaton. If $c \gtrsim \mathcal{O}(10)$, the constraint on the Hubble parameter can be relaxed further, making the chaotic inflation consistent with the axion dark matter.

So far we have focused on the case of $m_{a}>H_{\text {inf }}$. If $m_{a}<H_{\mathrm{inf}}$, the axion acquires quantum fluctuations. It is, however, possible that the axion remains heavy for a while even after inflation, and starts to oscillate and decays into photons. Then the isocurvature constraint can be avoided. To this end, one needs to delay the commencement of oscillation of $\phi$ as well as thermalization of the SM particles. The former requires a rather flat potential of $\phi$ at large field values, while the latter is possible if the inflaton decays into hidden particles, not into the SM particles.

Lastly let us mention the cosmological evolution after inflation. The Universe will be reheated by the decay of either the inflaton or $\phi$. In the latter case, the preheating as well as subsequent dissipation effects will be important if $\phi$ passes through the origin during oscillations [25, 26].

In this letter we have proposed a novel mechanism to suppress the axion CDM isocurvature perturbations. The point is that, if QCD becomes strong at an intermediate or high scale during inflation, the axion can be so heavy that no sizable quantum fluctuations at superhorizon scales are produced.

\section{Acknowledgment}

This work was supported by Grant-in-Aid for Scientific Research (C) (No. 23540283) [KSJ], Scientific Research on Innovative Areas (No.24111702 [FT], No. 21111006 [FT], and No.23104008 [KSJ and FT]), Scientific Research (A) (No. 22244030 and No.21244033) [FT], and JSPS Grant-in-Aid for Young Scientists (B) (No. 24740135) [FT].

[1] R. D. Peccei and H. R. Quinn, Phys. Rev. Lett. 38, 1440 (1977); Phys. Rev. D 16, 1791 (1977).

[2] For a review, see J. E. Kim, Phys. Rept. 150, 1 (1987); H. Y. Cheng, Phys. Rept. 158, 1 (1988); J. E. Kim and G. Carosi, Rev. Mod. Phys. 82, 557 (2010); A. Ringwald, Phys. Dark Univ. 1 (2012) 116; M. Kawasaki and K. Nakayama, arXiv:1301.1123 [hep-ph].

[3] P. A. R. Ade et al. [ Planck Collaboration], arXiv:1303.5082 [astro-ph.CO].

[4] D. Seckel and M. S. Turner, Phys. Rev. D 32, 3178 (1985).

[5] D. H. Lyth, Phys. Lett. B 236, 408 (1990).

[6] D. H. Lyth, Phys. Rev. D 45, 3394 (1992).

[7] T. Kobayashi, R. Kurematsu and F. Takahashi, JCAP 1309, 032 (2013) arXiv:1304.0922 [hep$\mathrm{ph}]$.

[8] A. D. Linde, Phys. Lett. B 129, 177 (1983).

[9] M. Kawasaki, M. Yamaguchi and T. Yanagida, Phys. Rev. Lett. 85, 3572 (2000). 
[10] R. Kallosh and A. Linde, JCAP 1011, 011 (2010).

[11] K. Nakayama, F. Takahashi and T. T. Yanagida, Phys. Lett. B 725, 111 (2013) arXiv:1303.7315 [hep-ph]]; JCAP 1308, $038 \quad$ (2013) arXiv:1305.5099, arXiv:1305.5099 [hep-ph]].

[12] A. D. Linde et al, Phys. Lett. B 246, 353 (1990).

[13] D. H. Lyth et al, Phys. Lett. B 283, 189 (1992).

[14] A. D. Linde, Phys. Lett. B 259, 38 (1991).

[15] S. Folkerts, C. Germani and J. Redondo, arXiv:1304.7270 [hep-ph].

[16] G. R. Dvali, hep-ph/9505253.

[17] T. Banks and M. Dine, Nucl. Phys. B 505, 445 (1997).

[18] K. Choi, H. B. Kim and J. E. Kim, Nucl. Phys. B 490, 349 (1997).

[19] I. Affleck and M. Dine, Nucl. Phys. B 249, 361 (1985).

[20] F. L. Bezrukov and M. Shaposhnikov, Phys. Lett.
B 659, 703 (2008).

[21] K. Nakayama and F. Takahashi, JCAP 1211, 007 (2012).

[22] G. F. Giudice and R. Rattazzi, Nucl. Phys. B 511, 25 (1998); N. Arkani-Hamed et al, Phys. Rev. D 58, 115005 (1998).

[23] I. Affleck, M. Dine and N. Seiberg, Nucl. Phys. B 241, 493 (1984).

[24] K. S. Jeong and F. Takahashi, JHEP 1208, 017 (2012) arXiv:1201.4816 [hep-ph]]; K. S. Jeong and F. Takahashi, JHEP 1304, 121 (2013) arXiv:1302.1486 [hep-ph]].

[25] J. Garcia-Bellido, D. G. Figueroa and J. Rubio, Phys. Rev. D 79, 063531 (2009).

[26] K. Mukaida and K. Nakayama, JCAP 1303, 002 (2013). 